\title{
Model of Driver's Eye Movement and ECG Index under Tunnel Environment Based on Spatiotemporal Data
}

\author{
Weiwei Qi $\left(D^{1}{ }^{1}\right.$ Bin Shen $\left(D,{ }^{1}\right.$ and Linhong Wang ${ }^{2}{ }^{2}$ \\ ${ }^{1}$ School of Civil Engineering and Transportation, South China University of Technology, Guangzhou, Guangdong 510641, China \\ ${ }^{2}$ School of Transportation, Jilin University, Changchun, Jilin 130012, China \\ Correspondence should be addressed to Linhong Wang; wanghonglin0520@126.com
}

Received 17 October 2019; Revised 7 December 2019; Accepted 18 January 2020; Published 17 February 2020

Academic Editor: Jaeyoung Lee

Copyright (c) 2020 Weiwei Qi et al. This is an open access article distributed under the Creative Commons Attribution License, which permits unrestricted use, distribution, and reproduction in any medium, provided the original work is properly cited.

In order to improve the driver's physiological and psychological state, the driver's mental load which is caused by sight distance, lighting, and other factors in the tunnel environment should be quantified via modeling the spatiotemporal data. The experimental schemes have been scientifically designed based on methods of traffic engineering and human factor engineering, which aims to test the driver's spatiotemporal data of eye movement and ECG (electrocardiogram) index in the tunnel environment. Firstly, the changes in the driver's spatiotemporal data are analyzed to judge the changing trend of the driver's workload in the tunnel environment. The results show that the cubic spline interpolation function model can fit the dynamic changes of average pupil diameter and heart rate (HR) growth rate well, and the goodness of fit for the model group is above 0.95. So, tunnel environment makes the driver's typical physiological indicators fluctuate in the coordinates of time and space, which can be modeled and quantified. Secondly, in order to analyze the classification of tunnel risk level, a fusion model has been built based on the functions of average pupil diameter and HR growth rate. The tunnel environmental risk level has been divided into four levels via the fusion model, which can provide a guidance for the classification of tunnel risk level. Furthermore, the fusion model allows tunnel design and construction personnel to adopt different safety design measures for different risk levels, and this method can effectively improve the economy of tunnel operating safety design.

\section{Introduction}

Tunnel is a typical bad visual environment, and driving in tunnel environment is a relatively dangerous activity. Amundsen and Ranes analyzed the traffic accident data in Norway and pointed out that the accident severity in the tunnel is higher than that in the highway [1]. A survey from Italy shows that severe accident rates and cost rates in tunnels were higher than those on the corresponding motorways [2]. Driving performance in tunnels is different from freeway driving, and darker lighting conditions and enclosed space will make drivers nervous and increase the effort required to maintain lateral control of the vehicle, which will affect drivers' psychological state and driving behavior [3, 4]. Related research shows that visual intervention is an effective method in vehicle trajectories' intervention $[5,6]$, and the tunnel safety can be improved by using visual intervention method to affect the driver's driving behavior in tunnel section. Therefore, it is of practical significance to study the driver's physiological and psychological state in tunnel environment, which reduces the accident rate in tunnel environment.

There are many factors that affect the safety of tunnel driving: road alignment, transition of antisliding performance, traffic states, and differences in internal and external environments [7]. In recent years, scholars have carried out lots of research about the highway traffic safety to improve the driving safety, especially for the tunnel environment $[8,9]$. Manser and Hancock studied how the type of visual pattern and presence of texture applied to transportation tunnel walls differentially affected driving performance based on simulated driving experiment [10]. Meng et al. proposed a novel quantitative risk assessment model to assess the risks in the nonhomogeneous urban road tunnels 
in 2011, and the inverse Gaussian regression model had been used to estimate the rear-end vehicle crash frequency in road tunnels; then the relationship between the time to collision and its contributing factors had been establish in 2012 [11-13]. Calvi and D'Amico analyzed the driver's speed characteristics and speed control in tunnel environment based on simulated driving [14]. Rudin-Brown et al. explored the influence of mobile phone use on driving safety in the tunnel based on the method of simulated driving [15]. Kircher and Ahlstrom investigated the impact of tunnel illumination and design on driving performance; then Kircher and Ahlstrom discovered that the driver's attention had a higher impact on performance than tunnel design [16]. Moretti et al. proposed a life-cycle cost analysis method to maximize energy saving and road safety via LED (LightEmitting Diode) technology [17, 18].

Scientific research on the driver's physiology and psychology began in the early nineteenth century. In recent years, with the improvement of medical research and the use of advanced instruments, scholars have continuously explored the integration of physiology and traffic engineering $[19,20]$. Many scholars have studied the physiological and psychological characteristics of drivers in tunnel environment, which can provide experience for the selection of indicators and data analysis in this paper. Cho et al. calibrated the threshold of illumination intensity in tunnels based on the driver's visual characteristics [21]. He et al. recorded the eye movement parameters of drivers passing through the tunnel and analyzed the influence of tunnel lighting environment on driving safety [22]. Feng et al. studied changes in physiological and behavioral characteristics in longitudinal segments of urban underpass tunnels by conducting a real-vehicle experiment [23]. Kening et al. conducted the real-vehicle experiment with participants under different tunnels, in order to study the driver mental workload variation in exit of super long tunnel on expressway [24]. Chen et al. carried out a driving simulator experiment based on a box truck module, in order to investigate the safety of the truck under crosswind at the bridge-tunnel section [25]. It can be seen that eye movement index, electrocardiogram index, electroencephalogram index, workload, and mental load are commonly used by scholars [26, 27]. Because the tunnel environment mainly affects the driver's vision and the enclosed space will bring tension and anxiety, this paper mainly chooses two indicators of eye movement and electrocardiogram.

At present, the research of the driver safety in tunnel environment mainly focuses on the analysis of the driver's speed, vision, and ECG characteristics in tunnel environment. On the basis of scientific experiment design and data acquisition, scholars use statistical methods to analyze the distribution of the driver's physiological and psychological indicators in tunnel environment, which provides a reference for the experimental design and statistical analysis of this paper. However, the existing research cannot reflect the temporal and spatial distribution of drivers' physiological and psychological state in tunnel environment and cannot evaluate the risk level of different road section in tunnel environment. Thus, this paper aims to explore the spatiotemporal distribution of driver's physiological and psychological indicators and study the classification of the risk level in tunnel environment. Firstly, the driver's spatiotemporal data of eye movement and ECG index are tested in the tunnel environment. Secondly, models of average pupil diameter and HR growth rate change trend are constructed in different spatial nodes of tunnel. Lastly, a classification model of tunnel risk level is constructed to effectively evaluate the risk level of different road sections in tunnel environment.

The remainder of this paper is organized as follows. Section 2 describes the methodology of the simulated driving experiment. Section 3 gives some experimental results for eye movement characteristics and ECG index. Then, models of the driver's average pupil diameter and HR rate are illustrated in Section 4, and the classification model of tunnel risk level is also achieved. Finally, this paper ends with some conclusions in Section 5.

\section{Method}

2.1. Participant. A total of 31 participants participated in the experiment; all drivers who are involved in the test hold a driver license and already have driving experience in the tunnel. Due to the age and gender of the test subjects' unbalanced distribution, the driver's age and gender differences are ignored during the process of data analysis.

2.2. Experimental Equipment and Scenario. The main experimental equipment of this experiment is as shown in Figure 1, including the six-degree-of-freedom traffic safety simulation driving device for constructing the simulated driving environment, MP36R physiological tester for collecting the driver's ECG index, SMI wireless glasses for collecting the driver's eye movement index, and other related auxiliary equipment, such as laptops, timers, etc.

The objective of this study is to investigate the temporal and spatial distribution of drivers' physiological and psychological indicators in tunnels. To this end, a common section of highway was designed according to the Technical Standard of Highway Engineering (2014). The test scenes are based on the two-lane straight highway with design speed of $80 \mathrm{~km} / \mathrm{h}$. The cross section of the highway comprises two 3.75-meter-wide lanes and two 2.5-meter-wide shoulders. The total length of the highway is $10.1 \mathrm{~km}$, including 3 tunnels, of which, each tunnel is $700 \mathrm{~m}$ long, and the interval between the two adjacent tunnels is $2 \mathrm{~km}$ (Figure 2). To ensure that the simulated environment is consistent with the real highway environment, there was some simulated vehicles moving in the same direction as the test vehicle, so that the traffic conditions are free-flow during the simulation. Trees, vertical speed limit signs, and traffic barriers were also added in the roadside to ensure drivers have the same feeling as real highway environment when driving in the test scenes. 

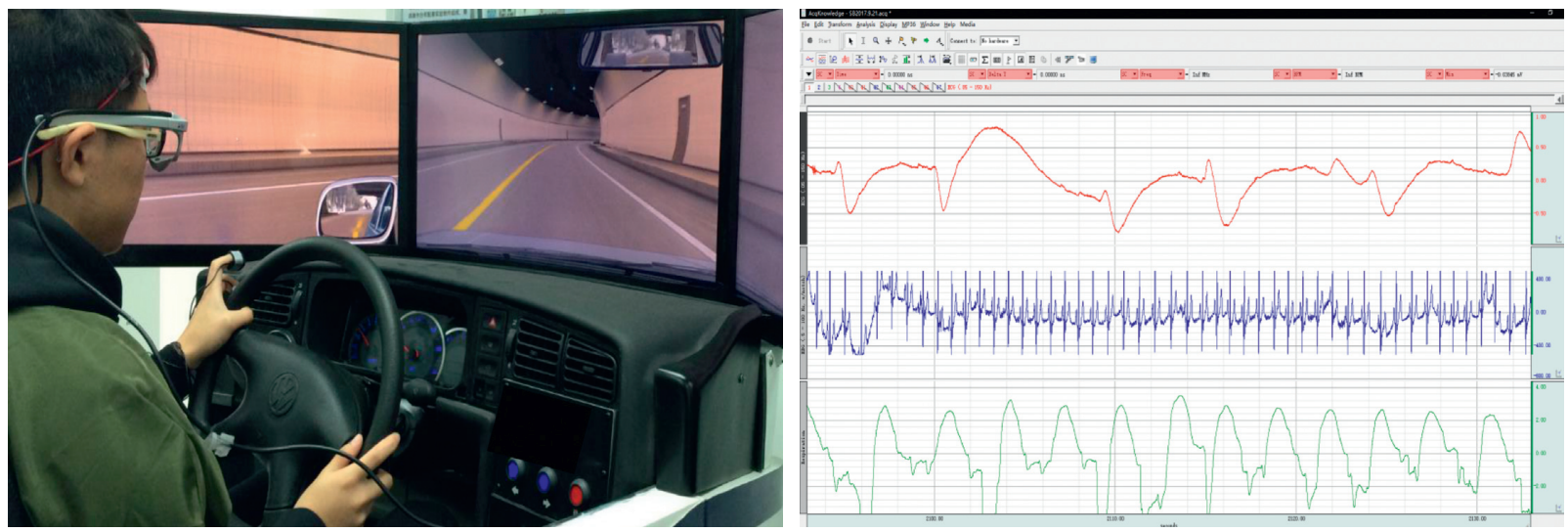

FIGURE 1: Main test equipment and data analysis interface.

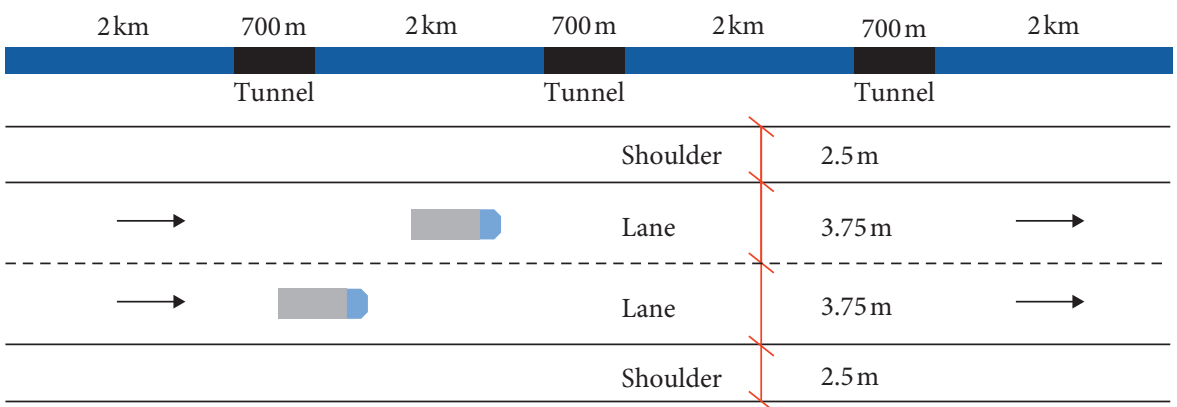

Figure 2: Experimental road conditions.

2.3. Experimental Procedure. The experiment has been carried out according to the following steps:

Step 1. The time of the experimental equipment has been checked to ensure that the time of all equipment is unified, and then a preliminary experiment has been conducted to ensure the reliability of the whole experiment.

Step 2. The SMI wireless glasses and the physiological instrument have been worn and calibrated for the test drivers. Then, the drivers have one hour to operate the driving stimulation to ensure the reliability of experimental data, so that drivers' strangeness on the simulated driving platform can be eliminated.

Step 3. The official experiment has been carried out, drivers in the test run normally according to the experimental design route, and the start time of the vehicle was used as the time base point to record the arrival and departure time of the driver in each tunnel section.

Step 4. The experimental data has been saved and the validity of the data has been checked after the experiment. Then, all the drivers completed the experiment according to the process strictly.

2.4. Data Collection. According to the experimental results, eye movement and ECG data of different drivers fluctuates greatly when the driver passes the first tunnel and the third tunnel, due to that different drivers have different adaptability to the simulated driving scenario. Some drivers have not adapted to the simulated driving platform fully during the first tunnel, and some drivers have adapted to the environmental parameters of the tunnel during the third tunnel. Therefore, the following research is based on the eye movement and ECG data of the second tunnel.

According to the switching time of the experimental scene recorded by the experimenter, the eye movement data analysis software can directly derive the fixation duration, the average pupil diameter, the blink duration, et al. Part of the eye movement data during the second tunnel is shown in Table 1.

Similar to eye movement data, the heart rate (HR) value during the second tunnel can be directly derived from the physiological data analysis software. Because of the individual differences between drivers, simply analyzing for the HR values is likely to cause large errors. Therefore, this paper uses the driver's HR growth rate for analysis and modeling. The calculation formula for heart rate indicators is shown in (1); the HR value and HR growth rate data is shown in Table 2.

$$
N_{i}=\frac{n_{i}-n_{p}}{n_{p}} \times 100 \% .
$$

In this formula, $N_{i}$ indicates the driver's heart rate at a certain moment, $n_{i}$ indicates the HR value of the driver at a certain moment, and $n_{p}$ indicates the average $\mathrm{HR}$ value of the driver in a calm state. 
TABLe 1: Eye movement data during the second tunnel.

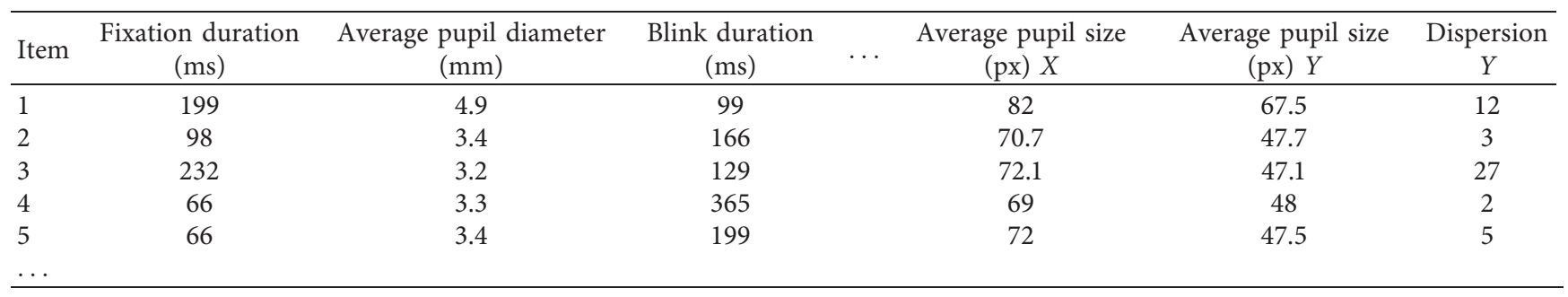

TABLE 2: Data of the HR value and HR growth rate.

\begin{tabular}{lcccccc}
\hline & \multicolumn{2}{c}{ Participant 1 } & \multicolumn{2}{c}{ Participant 2 } & \multicolumn{2}{c}{ Participant 31 } \\
& Heart rate & HR growth rate & Heart rate & HR growth rate & Heart rate & HR growth rate \\
\hline 1 & 103.448 & 0.293 & 85.714 & 0.143 & 90.634 & 88.757 \\
2 & 101.351 & 0.267 & 87.977 & 0.173 & 87.719 & 0.183 \\
3 & 100.334 & 0.254 & 84.746 & 0.130 & 81.967 & 0.170 \\
4 & 99.338 & 0.242 & 79.576 & 0.061 & \\
$\ldots$ & & & & & 0.093 \\
\hline
\end{tabular}

\section{Results}

3.1. Analysis of Eye Movement Characteristics. As shown in Table 3, the eye movement data of the driver in the tunnel environment is counted within a time window of $30 \mathrm{~s}$. By comparing the driving concentration and mental load of drivers in the tunnel and normal driving environment, it is found that the number of blinks of the driver in the tunnel is significantly smaller than the number of blinks in the normal environment $(P<0.05)$, and the average blink time in the tunnel (306.93 $\mathrm{ms}$ ) is also smaller than the average blink time in the normal environment $(323.87 \mathrm{~ms})$, which indicates that drivers are more attentive and have a higher mental load in the tunnels due to the dim light and poor visual distance. In addition, the numbers of gazes and scans in the tunnel are significantly less than those of driving in normal situations $(P<0.01)$, while the average gaze time is higher than normal ( $219.60 \mathrm{~ms})$. There is no significant difference in average scan time between the two groups (the tunnel is $79.79 \mathrm{~ms}$, normal $80.24 \mathrm{~ms}$ ), which indicates that the driving environment in the tunnel is more monotonous than in the normal environment [22], and drivers need to observe the surroundings more frequently in the normal environment than in the tunnel environment, and their sight will move more frequently.

The pupil diameter is an important indicator of the driver's sensitivity to light source [22]. The size of the pupil is affected by the illuminance: the larger the illuminance value, the smaller the pupil diameter. The average pupil diameter data is selected from the $60 \mathrm{~s}$ before entering the tunnel to the $60 \mathrm{~s}$ after leaving the tunnel. The result is shown in Figure 3.

According to Figure 3, drivers need to be more focused in the tunnel because of the poor sight, and the pupil diameter is larger than the pupil diameter in the normal environment. During the process of entering tunnel, the diameter of the driver's pupil shows a trend of gradual increase. The driver's concentration and mental load are increasing. During the process of leaving the tunnel, the diameter of the driver's pupil
TABle 3: Analysis of the driver's eye movement data in the tunnel environment.

\begin{tabular}{|c|c|c|c|c|c|}
\hline Items & & Mean & $\mathrm{Sd}$ & $T$ & $P$ \\
\hline Blink count & $\begin{array}{c}\text { Tunnel } \\
\text { Freeway }\end{array}$ & $\begin{array}{l}12.00 \\
22.29\end{array}$ & $\begin{array}{c}7.979 \\
10.193\end{array}$ & -2.10 & 0.049 \\
\hline $\begin{array}{l}\text { Blink duration average } \\
(\mathrm{ms})\end{array}$ & $\begin{array}{c}\text { Tunnel } \\
\text { Freeway }\end{array}$ & $\begin{array}{l}306.93 \\
323.87 \\
\end{array}$ & $\begin{array}{c}106.464 \\
41.923 \\
\end{array}$ & -0.39 & 0.706 \\
\hline Fixation count & $\begin{array}{l}\text { Tunnel } \\
\text { Freeway }\end{array}$ & $\begin{array}{l}37.86 \\
72.86\end{array}$ & $\begin{array}{l}11.423 \\
11.452\end{array}$ & -5.73 & 0.000 \\
\hline $\begin{array}{l}\text { Fixation duration } \\
\text { average }(\mathrm{ms})\end{array}$ & $\begin{array}{l}\text { Tunnel } \\
\text { Freeway }\end{array}$ & $\begin{array}{l}227.51 \\
219.60\end{array}$ & $\begin{array}{c}103.510 \\
59.490\end{array}$ & 0.18 & 0.864 \\
\hline Saccade count & $\begin{array}{c}\text { Tunnel } \\
\text { Freeway }\end{array}$ & $\begin{array}{l}36.00 \\
71.14\end{array}$ & $\begin{array}{l}14.900 \\
19.308\end{array}$ & -3.81 & 0.003 \\
\hline $\begin{array}{l}\text { Saccade duration } \\
\text { average }(\mathrm{ms})\end{array}$ & $\begin{array}{l}\text { Tunnel } \\
\text { Freeway }\end{array}$ & $\begin{array}{l}79.79 \\
80.24\end{array}$ & $\begin{array}{l}4.591 \\
5.930\end{array}$ & -0.16 & 0.875 \\
\hline
\end{tabular}

shows a trend of sharp decrease due to the sudden increase of light. At the same time, the driver's line of sight is severely obstructed, and appropriate measures (such as reducing the speed) are needed to prevent accidents. After leaving the tunnel, the diameter of the driver's pupil gradually rises and returns to the normal level.

\subsection{Analysis of Driver's ECG Index in the Tunnel Environment}

3.2.1. The Driver's HR Growth Rate. The HR growth rate from the $60 \mathrm{~s}$ before entering the tunnel to the $60 \mathrm{~s}$ after leaving the tunnel is selected as the analysis data. The result is shown in Figure 4.

Previous studies have shown that the change in heart rate is a direct reflection of the driver's psychological tension. The changes of HR growth rate indicate that the driver's psychological tension is tight, which will lead to the driver's misjudgment and misoperation, thus causing traffic accidents. From Figure 4, we can see that the driver's HR growth rate increases in the tunnel section ranging from $12 \%$ to $27 \%$; the HR growth rate in the normal section is maintained at a relatively stable level. When the driver begins to enter 


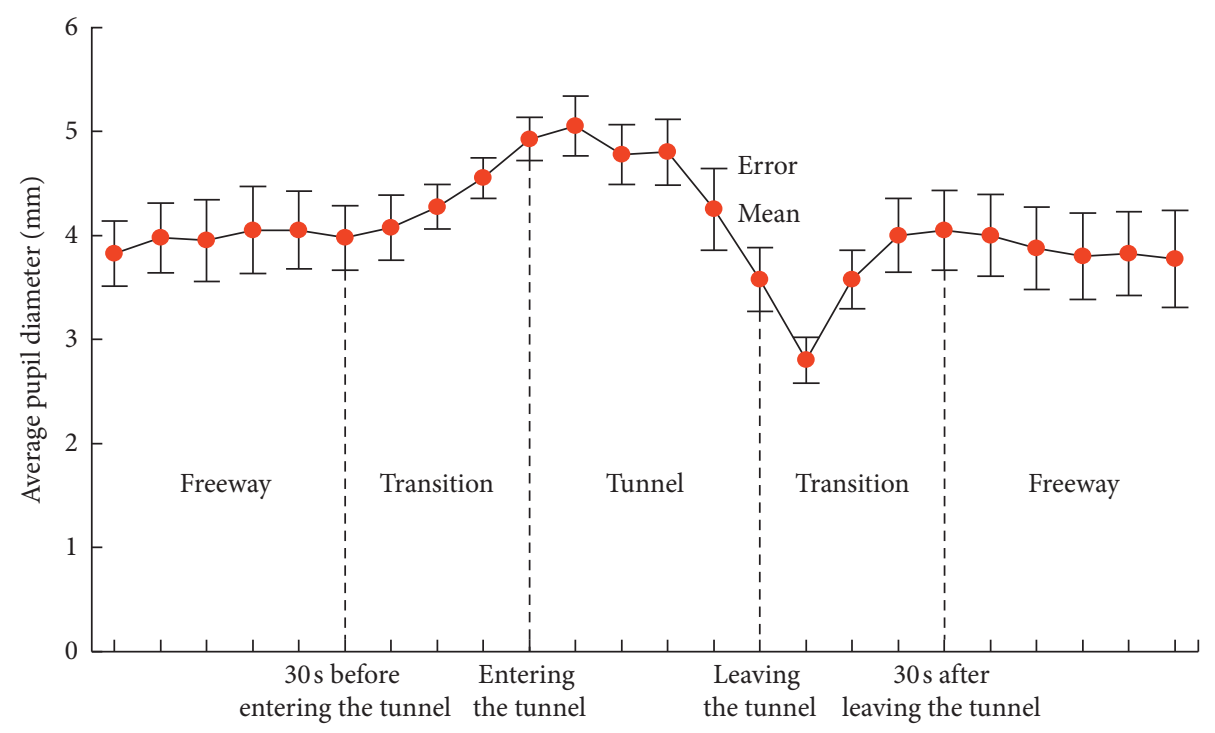

FIgURE 3: Changes in average pupil diameter during driving through a tunnel.

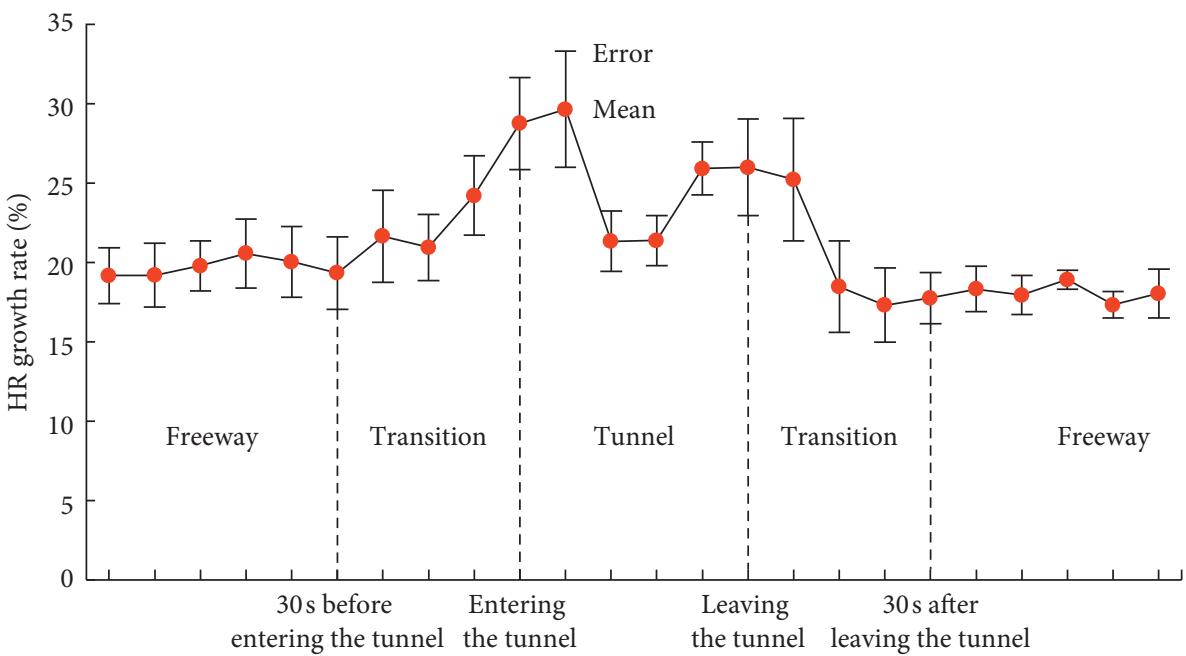

FIGURE 4: Changes of HR growth rate during driving through a tunnel.

the tunnel, the driver's HR growth rate increases. The driver's heart rate is maintained at a certain level in the tunnel. When the driver is about to leave the tunnel, the driver's heart rate rises. As the vehicle leaves the tunnel, the driver's heart rate gradually returns to a stable value. It is worth noting that the driver's heart rate does not suddenly become stable after entering the tunnel; it is a gradual process. The main reason for the change in the driver's heart rate during the process of passing the tunnel is the sudden change of sight distance and lighting conditions. After vehicle enters the tunnel, the driver gradually adapts to the sight distance and lighting conditions inside the tunnel, so the driver's heart rate gradually changes to a stable level.

3.2.2. Heart Rate Variability. HRV (heart rate variability) is a method that has been widely used in the academic literature. It reflects the state of ANS (autonomic nervous system) [28]. It is therefore a good objective tool for assessing emotional responses [29]. There are a number of different measurements which are derived from the interval between heartbeats (RR).

Time-domain analysis of HRV mainly included the following:

(i) MEAN: mean value of the RR intervals

(ii) SDNN: standard deviation of the RR intervals

(iii) RMSSD: root mean square of successive differences

(iv) SDSD: standard deviation of difference between adjacent RR intervals.

Frequency-domain analysis of HRV mainly included the following:

(i) HF: high frequency

(ii) LF: low frequency

(iii) LF/HF: the low frequency and high frequency ratio. 
The driver's heart is innervated by both sympathetic and vagal nerves. Under normal conditions, the excitability of vagus nerve is dominant. Under fatigue, excitation, and tension, sympathetic nerve is dominant. In terms of timedomain index, according to the data in Table 3, the average MEAN of RR interval of drivers decreases after entering the tunnel section; that is, the number of inner jumps of drivers in the tunnel section increases, which indicates that drivers have a certain degree of tension because of the bad visual distance. In the tunnel section, the SDNN index value of drivers increased, which indicates that the sympathetic nerve activity increases, the parasympathetic nerve activity decreases, and the mental load increases. The trend of RMSSD and SDSD is the same as that of SDNN. After entering the tunnel, drivers are affected by bad sight distance and the index values increases.

In terms of frequency-domain indicators, according to the data in Table 4, after the driver entered the tunnel section, the driver's LF value increases and the HF value decreases due to the deterioration of the driving environment, indicating that the driver's vagus nerve activity is weakened during this process, sympathetic activity is enhanced, the driver's mental condition is increasingly unstable, and physiological indicators vary greatly. On the one hand, because the visibility of the road is low in this environment, the driver's line of sight is smaller than the safe distance of the vehicle, and the driver needs to constantly adjust the speed and the nerves are tight. On the other hand, in order to prevent the driver from colliding with other vehicles, it is necessary to concentrate, and therefore the tunnel conditions have greater mental stress on the driver.

\section{Modeling}

4.1. Driver's Average Pupil Diameter Modeling. The tunnel environment has a significant impact on the driver's eye movement and ECG indicators. In order to quantify the trend of this influence, the variation of the average pupil diameter has been obtained by the interpolation method. First, a certain number of data points are selected from the driving process from 30 seconds before entering the tunnel to 30 seconds after leaving the tunnel. Then the interpolation function of the driving process is obtained by using cubic spline interpolation method, and the function $f(x)$ is drawn with the help of MATLAB, as shown in Figure 5(a). According to the different functional areas of the tunnel, the function is divided into three stages: before entering the tunnel $\left(f_{1}\right)$, inside the tunnel $\left(f_{2}\right)$, and after leaving the tunnel $\left(f_{3}\right)$; time axes of functions $f_{1}$ and $f_{2}$ are coordinate origins at the start of entering the tunnel, and the time axis of function $f_{3}$ is coordinate origins at the start of leaving the tunnel.

The fitted models are shown in (2)-(4). The R-square and RMSE of each model are shown in Table 5. The results of the cubic spline interpolation function fitting of each model are shown in Figures 5(b)-5(d). According to Table 4 and Figure 5 , it can be clearly seen that the $R$-square value of the model is greater than 0.95 , and the RMSE values are all less
TABle 4: Time- and frequency-domain indicators of the driver's heart rate variability.

\begin{tabular}{lcccc}
\hline \multirow{2}{*}{ Items } & \multicolumn{2}{c}{ Freeway } & \multicolumn{2}{c}{ Tunnel } \\
& Mean & Sd & Mean & Sd \\
\hline MEAN & 0.732 & 0.116 & 0.683 & 0.112 \\
SDNN & 0.052 & 0.043 & 0.067 & 0.076 \\
RMSSD & 0.062 & 0.092 & 0.082 & 0.101 \\
SDSD & 0.037 & 0.050 & 0.065 & 0.087 \\
HF & 0.821 & 0.095 & 0.797 & 0.078 \\
LF & 0.179 & 0.090 & 0.206 & 0.078 \\
LF/HF & 0.218 & 0.156 & 0.258 & 1.580 \\
\hline
\end{tabular}

than 0.1 , which satisfies the accuracy requirements in statistics, indicating that the goodness of fit of each model is higher.

$$
\begin{aligned}
f_{1}(x)= & 0.000757 x^{2}+0.05438 x+4.917 \\
f_{2}(x)= & 1.989 \exp \left(-\left(\frac{x-0.5788}{8.871}\right)^{2}\right) \\
& +4.802 \exp \left(-\left(\frac{x-20.14}{28.89}\right)^{2}\right) \\
f_{3}(x)= & 3.488-0.3046 \cos (0.1751 x) \\
& -0.5425 \sin (0.1751 x)
\end{aligned}
$$

4.2. Driver's HR Growth Rate Modeling. According to the ideas in part 4.1, the variation of the average pupil diameter has been obtained by the interpolation method, and the function $g(x)$ is drawn with the help of MATLAB as shown in Figure 6(a). According to the different functional areas of the tunnel, the image is divided into three stages: before entering the tunnel $\left(g_{1}\right)$, inside the tunnel $\left(g_{2}\right)$, and after leaving the tunnel $\left(g_{3}\right)$. The fitting model of the driver's HR growth rate in different functional sections of the tunnel is shown in (5)-(7). The $R$-square and RMSE of each model are shown in Table 6; the results of the cubic spline interpolation function fitting of each model are shown in Figures 6(b)-6(d). According to Table 6 and Figure 6 , it can be clearly seen that the $R$-square value of the model is greater than 0.99, and the RMSE values are all less than 0.5 , which satisfies the accuracy requirements in statistics, indicating that the goodness of fit of each model is higher.

$$
\begin{aligned}
g_{1}(x)= & 28.18 \exp \left(-\left(\frac{x-5.366}{24.84}\right)^{2}\right) \\
& +13.76 \exp \left(-\left(\frac{x+27.06}{11.38}\right)^{2}\right) \\
g_{2}(x)= & -0.0001704 x^{4}+0.01292 x^{3}-0.2877 x^{2}+1.432 x \\
& +27.98
\end{aligned}
$$




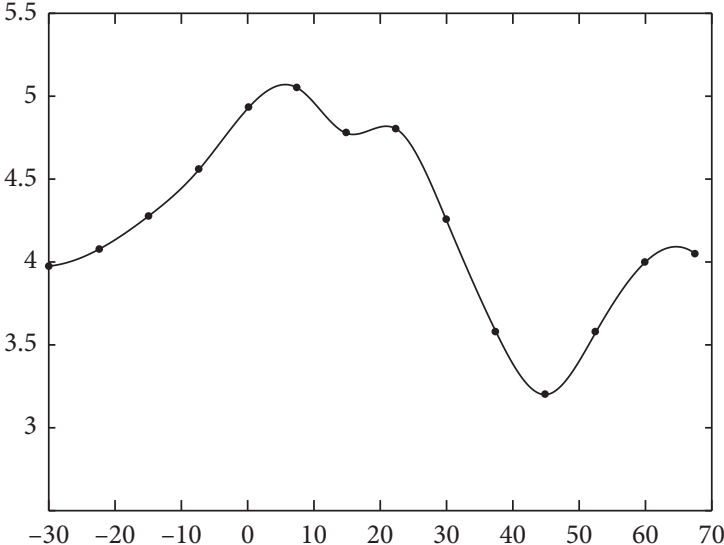

(a)

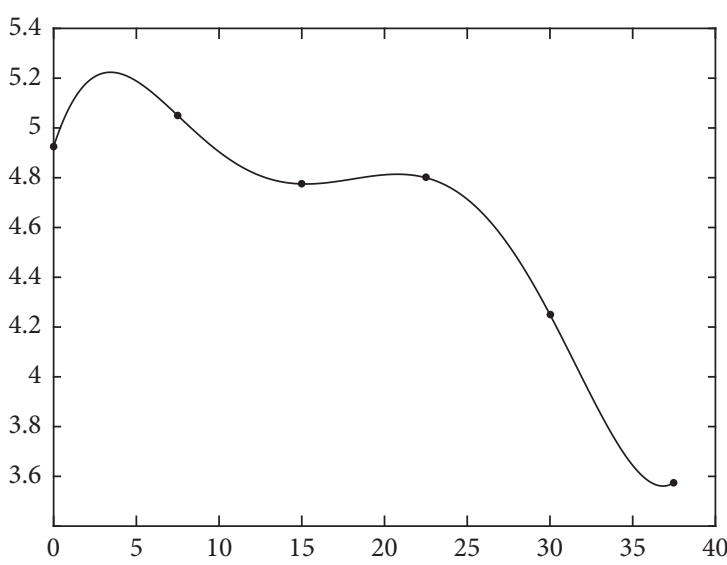

(c)

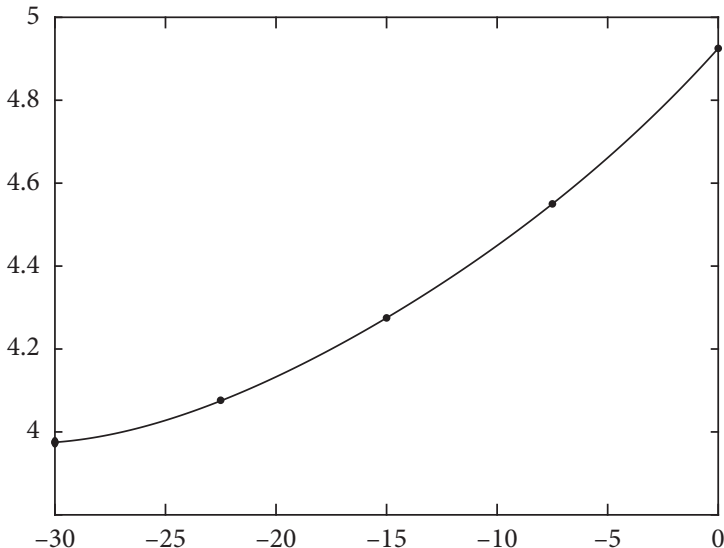

(b)

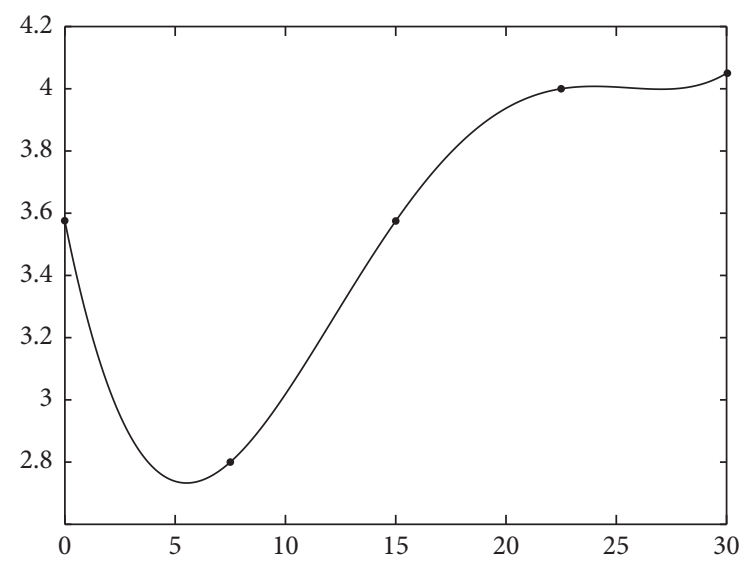

(d)

FIgURE 5: Piecewise cubic spline interpolation function model of average pupil diameter. (a) Piecewise cubic spline interpolation function. (b) Function $f_{1}$ fitting. (c) Function $f_{2}$ fitting. (d) Function $f_{3}$ fitting.

TABle 5: Evaluation index of the driver's average pupil diameter fitting model in tunnel environment.

\begin{tabular}{lccc}
\hline Evaluation index & $f_{1}$ & $f_{2}$ & $f_{3}$ \\
\hline$R$-square & 0.9999 & 0.9928 & 0.9578 \\
RMSE & 0.002563 & 0.04015 & 0.09991 \\
\hline
\end{tabular}

$$
\begin{aligned}
g_{3}(x)= & -1.985 e+04 \exp \left(-\left(\frac{x-14.1}{17.51}\right)^{2}\right) \\
& +1.986 e+0.4 \exp \left(-\left(\frac{x-14.09}{17.52}\right)^{2}\right) .
\end{aligned}
$$

4.3. Classification Model of Tunnel Risk Level. Tunnels are typical bad sight distance environments; driving in tunnels is very dangerous and easy to cause traffic accidents. In order to improve the driving safety in the tunnel environment, the tunnel design and construction personnel will optimize the safety of the tunnel, which needs so much of money and so many of material resources. Therefore, it is necessary to establish a classification model of tunnel risk level, which allows tunnel design and construction personnel to adopt different safety design measures for road sections on different risk levels. Tunnel manager can effectively improve the design economics of tunnel environmental safety.

In order to classify the risk level of the tunnel environment, a fusion model $T(x)$ needs to be built based on the functions $f(x)$ and $g(x)$. In the fusion model, $T(x)$ is the risk assessment value of tunnel environments, $\alpha$ is the weight coefficient of the driver's HR growth rate, $(1-\alpha)$ is the weight coefficient of the driver's average pupil diameter, $f^{\prime}(x)$ is the dimensionless form to $f(x)$, and $g^{\prime}(x)$ is the dimensionless form to $g(x)$. Then, the fusion model is shown as

$$
T(x)=\alpha f^{\prime}(x)+(1-\alpha) g^{\prime}(x)
$$

According to the research results in 4.1 and 4.2, the functions $f(x)$ and $g(x)$ need dimensional standardization. The models for dimensional standardization are shown as

$$
\begin{aligned}
f^{\prime}(x) & =\frac{f(x)-f(x)_{\max }}{f(x)_{\max }-f(x)_{\min }} \\
g^{\prime}(x) & =\frac{g(x)-g(x)_{\max }}{g(x)_{\max }-g(x)_{\min }} .
\end{aligned}
$$




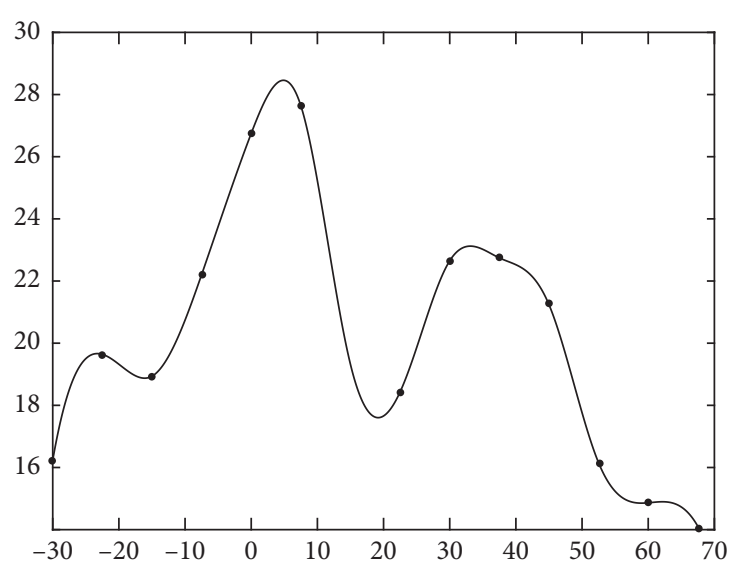

(a)

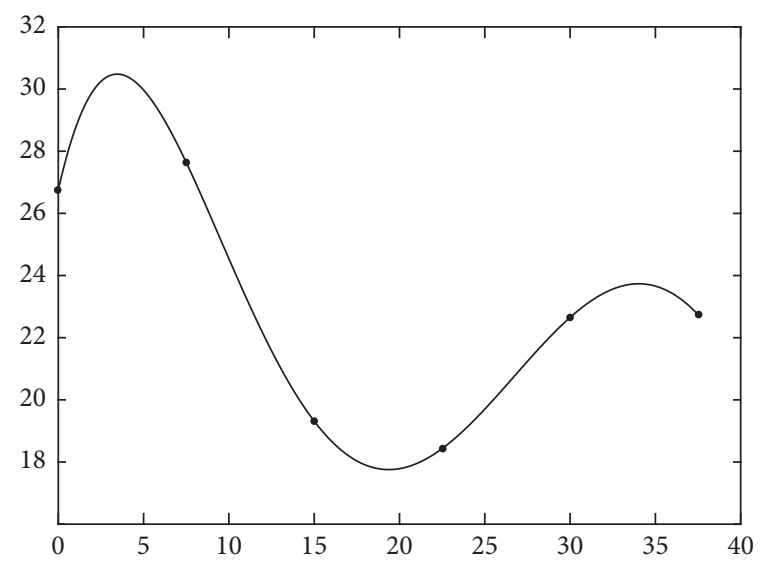

(c)

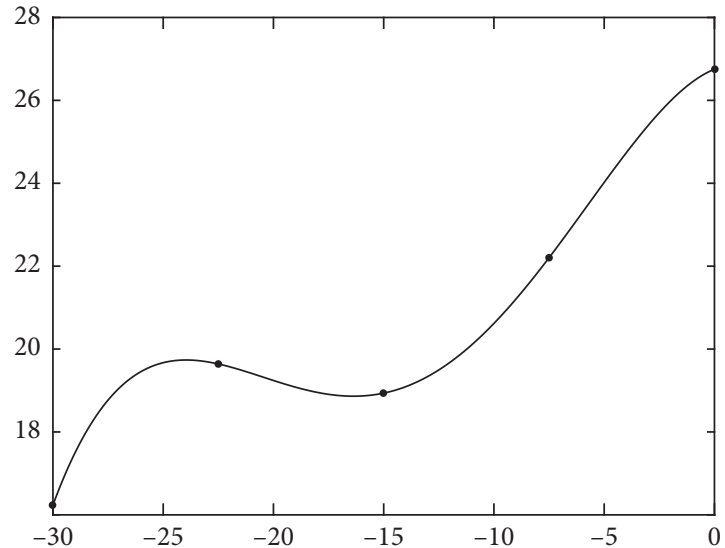

(b)

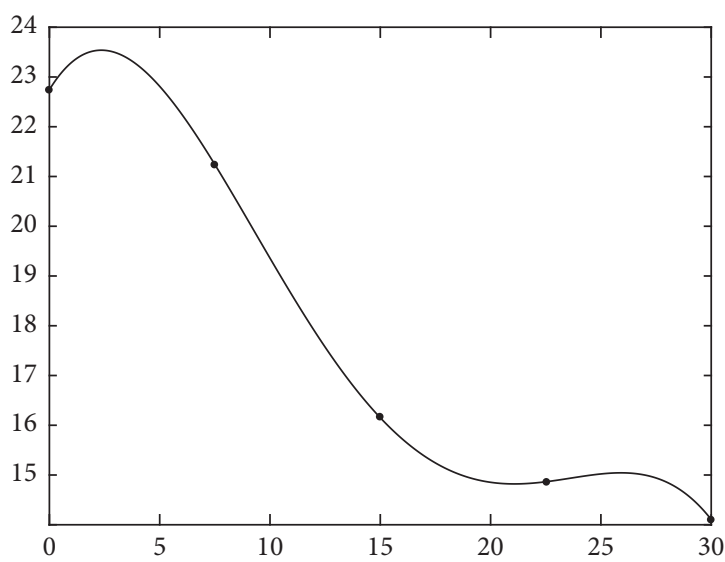

(d)

FIGURE 6: HR growth rate piecewise cubic spline interpolation function model. (a) Piecewise cubic spline interpolation function. (b) Function $g_{1}$ fitting. (c) Function $g_{2}$ fitting. (d) Function $g_{3}$ fitting.

TABLE 6: Evaluation index of the driver's HR growth rate fitting model in tunnel environment.

\begin{tabular}{lccc}
\hline Evaluation index & $g_{1}$ & $g_{2}$ & $g_{3}$ \\
\hline$R$-square & 0.9992 & 0.991 & 0.9996 \\
RMSE & 0.07524 & 0.3807 & 0.06458 \\
\hline
\end{tabular}

Based on (8), $T_{n}$ is defined to represent the risk assessment value of the common road section without tunnel or bridge, and the time interval $(a, b)$ is defined as the time for driving in the common road section without tunnel or bridge; then $T_{n}$ can be calculated by

$$
T_{n}=\frac{1}{b-a} \int_{a}^{b} T(x) \mathrm{d} x .
$$

The determination of the classification criteria of tunnel environmental risk level needs large amounts of traffic accident data, the experimental data that obtained in this study cannot be used to determine the classification criteria accurately, and there is no relevant research that has defined this criterion as well. Thus, for ease of use, the tunnel environmental risk level has been divided into four levels in this study, which are normal state, light danger, moderate danger, and severe danger. So, when the value of $T(x)$ is less than or equal to $T_{n}$, tunnel environmental risk level is normal; when the value of $T(x)$ is more than $T_{n}$ and less than or equal to $1.5 T_{n}$, tunnel environmental risk level is light danger; when the value of $T(x)$ is more than $1.5 T_{n}$ and less than or equal to $2 T_{n}$, tunnel environmental risk level is moderate danger; and when the value of $T(x)$ is more than $2 T_{n}$, tunnel environmental risk level is severe danger. The classification criteria of tunnel environmental risk level are shown in Table 7.

To verify the validity of the classification model, this research selects the road section of 30 seconds before entering the second tunnel to 30 seconds after leaving the second tunnel as the studied area. As there is no relevant research to prove the weight coefficient relationship between the driver's HR growth rate and the average pupil diameter in the tunnel environment at present, this paper takes $\alpha=0.5$ as an example to study the tunnel environment risk classification. $T_{n}$ can be calculated according to (10), and $T_{n}=0.7495$; the results are shown in Figure 7 .

As shown in Figure 7, the tunnel area can be divided into nine road sections according to classification model of tunnel risk level. Among the nine road sections, risk level of 
TABLE 7: Classification criteria of tunnel environmental risk level.

\begin{tabular}{lcccc}
\hline Level & Normal state & Light danger & Moderate danger & Severe danger \\
\hline Criterion & $T(x) \leq T_{n}$ & $T_{n}<T(x) \leq 1.5 T_{n}$ & $1.5 T_{n}<T(x) \leq 2 T_{n}$ & $T(x)>2 T_{n}$ \\
\hline
\end{tabular}

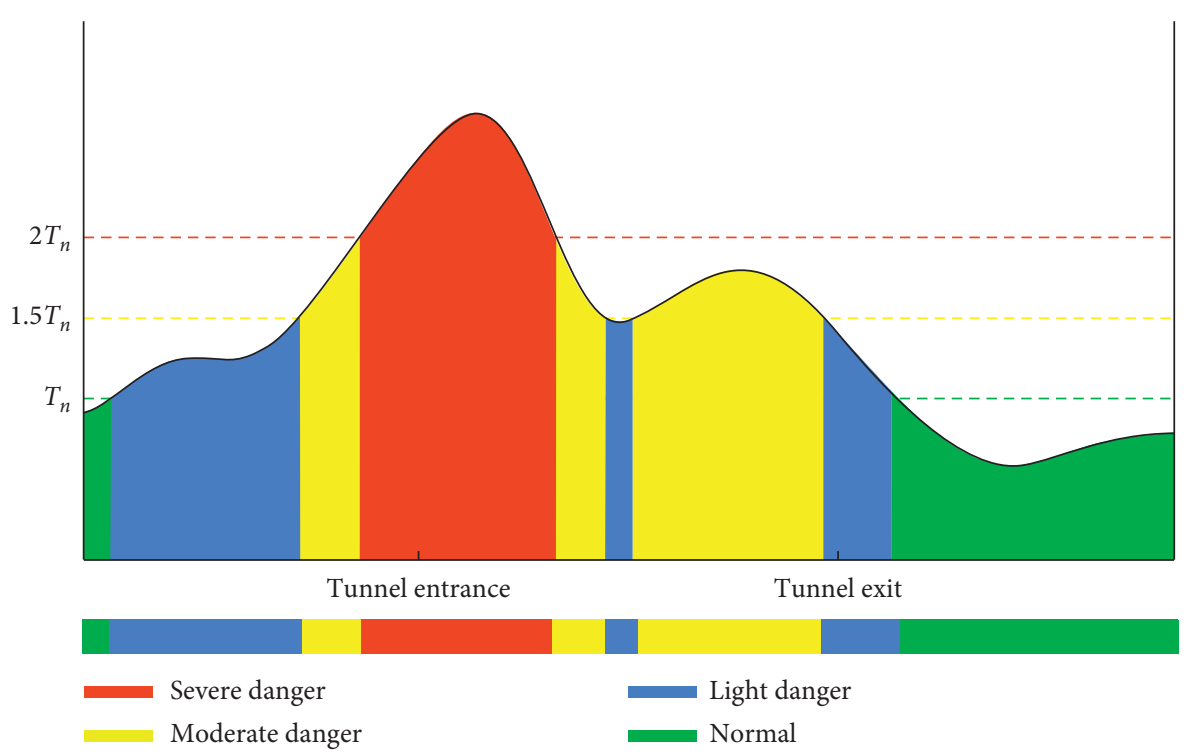

FIGURE 7: The results of tunnel environment risk level classification.

one road section is severe danger, risk level of three road sections is moderate danger, risk level of three road sections is light danger, and risk level of two road sections is normal. Compared with the total length of the tunnel area that has been studied in this paper, the lengths of road sections at different risk level are $17.8 \%$ (severe danger), $27.4 \%$ (moderate danger), $27.0 \%$ (light danger), and $27.8 \%$ (normal). So, if tunnel managers focus on optimizing road sections with risk level of severe danger (17.8\%), nearly $82.2 \%$ of the construction funds can be saved and the economic benefits in the tunnel construction can be greatly improved.

\section{Conclusions}

This paper has tested the eye movement and ECG indicators in the tunnel environment and constructed the cubic spline interpolation function modeling. The main conclusions are as follows:

(1) The driver's blinking times and average blinking time in tunnels are lower than the normal level, but the average fixation time is higher than the normal level. In the process of entering the tunnel, the driver's pupil diameter shows a trend of gradual increase, and in the process of leaving the tunnel, the driver's pupil diameter shows a trend of sharp decrease. Results show that the tunnel environment has a significant impact on the driver's eye movement index, with abnormal fluctuations in blinking times, average blinking time, and pupil diameter.

(2) The driver's HR growth rate changes significantly during the tunnel section, which ranges from $12 \%$ to $27 \%$. In the process of entering tunnel, the HR growth rate increases continuously and then maintains a relatively stable level in the tunnel. In the process of leaving the tunnel, the HR growth rate begins to decline and gradually returns to normal value. The results show that the tunnel environment also has a significant impact on the driver's heart rate index.

(3) The time-domain and frequency-domain indicators of the driver's heart rate variability fluctuate in varying degrees during driving in tunnels, among which the SDSD index changes obviously, and the maximum fluctuation range is $75.6 \%$, which indicates that the driver's mental load increases greatly in this environment and results in a strong sense of tension, panic, and fatigue.

(4) Based on the statistical analysis of the driver's eye movement and ECG index, the cubic spline interpolation function is introduced to accurately fit the dynamic trend of the driver's physiological index in the $30 \mathrm{~s}$ interval before and after entering and exiting the tunnel. The goodness of fit of each model is above 0.95 , which indicates that the impact of the tunnel environment on the driver's eye movement and ECG indicators can be quantified and predicted.

(5) A fusion model is constructed based on the models of the driver's average pupil diameter and HR growth rate to classify the risk level of the tunnel environment. According to this fusion model, the risk level of the tunnel environment can be divided into four levels, and the lengths of road sections with different risk level are $17.8 \%$ (severe danger), $27.4 \%$ (moderate danger), 27.0\% (light danger), and 27.8\% (normal). 
(6) The classification model of tunnel risk level can provide guidance for the classification of tunnel risk level, and tunnel personnel for design and construction can adopt different safety design measures for different risk levels, which can effectively improve the economy of tunnel operating safety design.

\section{Data Availability}

The driver's spatiotemporal data of eye movement and ECG (electrocardiogram) index in the tunnel environment that are used to support the findings of this study are currently under embargo, while the research findings are commercialized. Requests for data, 12 months after publication of this article, will be considered by the corresponding author.

\section{Disclosure}

The funders played no role in the design of the study or in the collection and interpretation of the data.

\section{Conflicts of Interest}

The authors declare no conflicts of interest.

\section{Acknowledgments}

This research was funded by the National Natural Science Foundation of China under grants numbers 71701070 and 71971097, the Science and Technology Project of Guangzhou City under grant number 201804010466, the Fundamental Research Funds for the Central Universities under grant number 2019MS120, Jilin Scientific and Technological Development Program under grant number 20180520180JH, and Jilin Education Planning Program under grant number JJKH20180149KJ.

\section{References}

[1] F. H. Amundsen and G. Ranes, "Studies on traffic accidents in Norwegian road tunnels," Tunnelling and Underground Space Technology, vol. 15, no. 1, pp. 3-11, 2000.

[2] C. Caliendo and M. L. De Guglielmo, "Accident rates in road tunnels and social cost evaluation," Procedia-Social and Behavioral Sciences, vol. 53, pp. 166-177, 2012.

[3] A. C. Beall and J. M. Loomis, "Visual control of steering without course information," Perception, vol. 25, no. 4, pp. 481-494, 1996.

[4] Y. Li and Y. Chen, "Driver vision based perception-response time prediction and assistance models on mountain highway curve," International Journal of Environmental Research and Public Health, vol. 14, no. 1, p. 31, 2016.

[5] G. Wu, F. Chen, X. Pan, M. Xu, and X. Zhu, "Using the visual intervention influence of pavement markings for rutting mitigation-part I: preliminary experiments and field tests," International Journal of Pavement Engineering, vol. 20, no. 6, pp. 734-746, 2019.

[6] X. Zhu, Z. Dai, F. Chen, X. Pan, and M. Xu, "Using the visual intervention influence of pavement marking for rutting mitigation II: visual intervention timing based on the finite element simulation," International Journal of Pavement Engineering, vol. 20, no. 5, pp. 573-584, 2019.

[7] S. Bassan, "Overview of traffic safety aspects and design in road tunnels," IATSS Research, vol. 40, no. 1, pp. 35-46, 2016.

[8] C. Xu, P. Liu, W. Wang, and Z. Li, "Evaluation of the impacts of traffic states on crash risks on freeways," Accident Analysis \& Prevention, vol. 47, pp. 162-171, 2012.

[9] H. Wen, J. Sun, Q. Zeng, X. Zhang, and Q. Yuan, "The effects of traffic composition on freeway crash frequency by injury severity: a Bayesian multivariate spatial modeling approach," Journal of Advanced Transportation, vol. 2018, Article ID 6964828, 7 pages, 2018.

[10] M. P. Manser and P. A. Hancock, "The influence of perceptual speed regulation on speed perception, choice, and control: tunnel wall characteristics and influences," Accident Analysis \& Prevention, vol. 39, no. 1, pp. 69-78, 2007.

[11] Q. Meng, X. Qu, X. Wang, V. Yuanita, and S. C. Wong, "Quantitative risk assessment modeling for nonhomogeneous urban road tunnels," Risk Analysis, vol. 31, no. 3, pp. 382-403, 2011.

[12] Y. Bie, X. Xiong, Y. Yan, and X. Qu, "Dynamic headway control for high-frequency bus line based on speed guidance and intersection signal adjustment," Computer-Aided Civil and Infrastructure Engineering, vol. 35, no. 1, pp. 4-25, 2020.

[13] Q. Meng and X. Qu, "Estimation of rear-end vehicle crash frequencies in urban road tunnels," Accident Analysis \& Prevention, vol. 48, pp. 254-263, 2012.

[14] A. Calvi and F. D'Amico, "A study of the effects of road tunnel on driver behavior and road safety using driving simulator," Advances in Transportation Studies, vol. 30, pp. 59-76, 2013.

[15] C. M. Rudin-Brown, K. L. Young, C. Patten, M. G. Lenné, and R. Ceci, "Driver distraction in an unusual environment: effects of text-messaging in tunnels," Accident Analysis \& Prevention, vol. 50, pp. 122-129, 2013.

[16] K. Kircher and C. Ahlstrom, "The impact of tunnel design and lighting on the performance of attentive and visually distracted drivers," Accident Analysis \& Prevention, vol. 47, pp. 153-161, 2012.

[17] L. Moretti, G. Cantisani, and P. Di Mascio, "Management of road tunnels: construction, maintenance and lighting costs," Tunnelling and Underground Space Technology, vol. 51, pp. 84-89, 2016.

[18] L. Moretti, G. Cantisani, P. Di Mascio, and S. Caro, “Technical and economic evaluation of lighting and pavement in Italian road tunnels," Tunnelling and Underground Space Technology, vol. 65, pp. 42-52, 2017.

[19] C. Ma, D. Yang, J. Zhou, Z. Feng, and Q. Yuan, "Risk riding behaviors of urban E-bikes: a literature review," International Journal of Environmental Research and Public Health, vol. 16, no. 13 , p. $2308,2019$.

[20] L. Zhang, B. Cui, M. Yang, F. Guo, and J. Wang, "Effect of using mobile phones on driver's control behavior based on naturalistic driving data," International Journal of Environmental Research and Public Health, vol. 16, no. 8, p. 1464, 2019.

[21] W. B. Cho, J. H. Jeong, D. G. Kim, and W. I. Park, "Comparison of safety level between driver's ages by threshold zone luminance level of vehicular traffic tunnel," Journal of the Korean Society of Road Engineers, vol. 17, no. 1, pp. 129-142, 2015.

[22] S. He, B. Liang, G. Pan, F. Wang, and L. Cui, "Influence of dynamic highway tunnel lighting environment on driving safety based on eye movement parameters of the driver," 
Tunnelling and Underground Space Technology, vol. 67, pp. 52-60, 2017.

[23] Z. Feng, M. Yang, W. Zhang, Y. Du, and H. Bai, "Effect of longitudinal slope of urban underpass tunnels on drivers' heart rate and speed: a study based on a real vehicle experiment," Tunnelling and Underground Space Technology, vol. 81 , pp. $525-533,2018$.

[24] Z. Kening and G. Bo, "Research on driver mental load in exit of super long tunnel," Technology \& Economy in Areas of Communications, vol. 19, no. 3, pp. 6-9, 2017.

[25] F. Chen, H. Peng, X. Ma, J. Liang, W. Hao, and X. Pan, "Examining the safety of trucks under crosswind at bridgetunnel section: a driving simulator study," Tunnelling and Underground Space Technology, vol. 92, Article ID 103034, 2019.

[26] E. Rendon-Velez, P. M. van Leeuwen, R. Happee, I. Horváth, W. F. van der Vegte, and J. C. F. de Winter, "The effects of time pressure on driver performance and physiological activity: a driving simulator study," Transportation Research Part F: Traffic Psychology and Behaviour, vol. 41, pp. 150-169, 2016.

[27] H. Wiberg, E. Nilsson, P. Lindén, B. Svanberg, and L. Poom, "Physiological responses related to moderate mental load during car driving in field conditions," Biological Psychology, vol. 108, pp. 115-125, 2015.

[28] K.-H. Choi, J. Kim, O. S. Kwon, M. J. Kim, Y. H. Ryu, and J.-E. Park, "Is heart rate variability (HRV) an adequate tool for evaluating human emotions?A focus on the use of the international affective picture system (IAPS)," Psychiatry Research, vol. 251, pp. 192-196, 2017.

[29] G. Valenza, P. Allegrini, A. Lanatà, and E. P. Scilingo, "Dominant Lyapunov exponent and approximate entropy in heart rate variability during emotional visual elicitation," Frontiers in Neuroengineering, vol. 5, no. 3, pp. 1-7, 2012. 